\title{
COMPETITIVIDAD AGROINDUSTRIAL EN EL CARMEN DE BOLÍVAR: ANÁLISIS DE CAPACIDADES PRODUCTIVAS SEGÚIN CLASES AGROLÓGICAS Y APTITUD DE SUELOS*
}

\author{
JULIO ADOLFO AMÉZQUITA LÓPEZ** \\ ALBERTO ENRIQUE GÓMEZ JUAN*** \\ GUSTAVO HERRERA SEBA**** \\ LEINYS MELGAREJO CAUSADO****
}

\begin{abstract}
RESUMEN
En los últimos años, la competitividad ha sido una temática muy debatida entre las instituciones encargadas de formular politicas públicas para el fomento del desarrollo económico, esto como consecuencia de la necesidad de incrementarla en los diferentes sectores productivos de las regiones. Para esta investigación se realizó un acercamiento al potencial agroindustrial de El Carmen de Bolívar de acuerdo al análisis de las capacidades productivas del municipio según las clases agrológicas y aptitud de suelos disponibles en la zona. Así mismo, se realizó un diagnostico de competitividad utilizando la metodología de las 5 fuerzas de Porter (Rivalidad entre productores, clientes, proveedores, sustitutos, competencia potencial).
\end{abstract}

Los resultados obtenidos permitieron identificar las implicaciones de las variaciones de uso en la mezcla de disposición de tierras para cultivos sobre el valor agregado y empleo generado por cada eslabón de la cadena.

\footnotetext{
*Este documento es producto del proyecto de investigación titulado "Análisis del Potencial Agroindustrial de la Producción Agrícola en El Carmen de Bolívar Bajo un Enfoque de Competitividad y Desarrollo Sostenible" realizado por el grupo de investigación Ciencia, Tecnología y Sociedad - CTS Unicartagena.

** Líder grupo de investigación Ciencia, Tecnología y Sociedad - CTS Unicartagena. Correo Electrónico: amezco@gmail.com.

*** Estudiante programa Ingeniería de Alimentos. Semillero Grupo de Investigación CTS Unicartagena.

**** Administradores Industriales Universidad de Cartagena. Grupo de Investigación CTS Unicartagena.
} 
Palabras Clave: Competitividad agroindustrial, producción agrícola, uso de suelo, clases agrológicas, aptitud de suelo.

\begin{abstract}
In recent years, competition has been a hotly debated topic among the institutions responsible for formulating public policies to promote economic development, that due to the need to increase in the productive sectors of the regions. For this investigation there carried out an approximation to the agroindustrial potential of Carmen of Bolivar of agreement to the analysis of the productive capacities of the municipality according to the agrologic classes and aptitude of available land in the area. Likewise, a diagnosis of competitiveness was realized using the methodology of 5 forces of Porter (Rivalry between producers, clients, suppliers, substitutes, potential competition).
\end{abstract}

The obtained results allowed to identify the implications of the variations of use in the mixture of disposition of lands for cultures on the added value and employment generated by every link of the chain.

Key Words: Agroindustrial competitiveness, agricultural production, land use, agrologic classes, aptitude of soil.

\title{
INTRODUCCIÓN
}

La producción agrícola, constituye una de las actividades más importantes en el proceso de apertura económica de las naciones. De modo tal, que es uno de los ejes centrales en las negociaciones entre los bloques comerciales. Así mismo, la agricultura está inherentemente ligada a dar solución a grandes problemas sociales, además de cumplir la función de preservar el ambiente y generar trabajo, jugando un papel fundamental en la defensa de un país debido a la ocupación del territorio y la reducción de la dependencia alimentaria (1).

El Carmen de Bolívar, ubicado en el departamento de Bolívar, es el municipio de mayor extensión en la Zona de Desarrollo Económico y Social (ZODES) Montes de María, así como el más habitado, con 954 km2 y 66.001 habitantes (34,42\% de la población de Montes de María). Esta característica lo convierte en una zona de importancia económica, en la cual se forjan dinámicas de producción y comercio agrícola que sostienen la seguridad alimentaria en el departamento. Sin embargo, actualmente el municipio atraviesa diversas problemáticas que lo limitan como centro 
de desarrollo económico. Entre ellos se destacan problemas como la precariedad institucional, expresada en la debilidad de la gestión pública, alto déficit fiscal, ausencia de planeación estratégica y bajo desarrollo organizacional, así como un incremento acelerado de la marginalidad y la pobreza, lo que trae consigo una vulnerabilidad creciente del capital humano y más de 125.000 hectáreas de tierra cultivable abandonadas por las familias desplazadas.

\section{ILUSTRACIÓN 1. UBICACIÓN GEOGRÁFICA DEL MUNICIPIO} DEL CARMEN DE BOLIIVAR

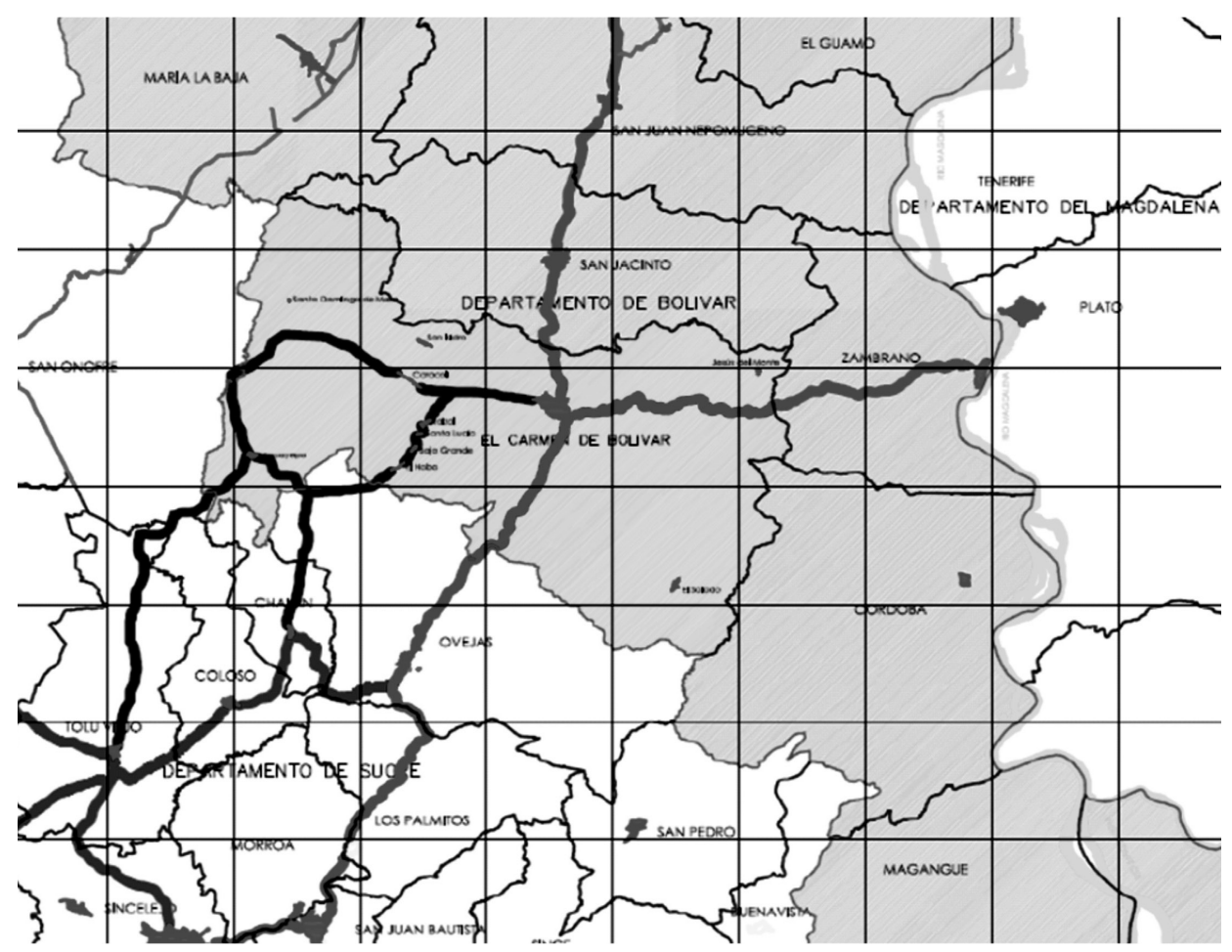

Fuente: Plan de ordenamiento Territorial del Carmen de Bolívar. Gobernación de Bolívar

Dadas estas circunstancias y en concordancia con previos estudios realizados por El Observatorio de Cultura, Política, Paz, Convivencia y Desarrollo de Los Montes de María, se realizó el presente estudio del potencial agroindustrial de El Carmen de Bolívar desde el punto de vista de competitividad y desarrollo sostenible.

Inicialmente se realizó un diagnóstico de competitividad de la producción agrícola y agroindustrial en el Carmen de Bolívar, utilizando la metodología de las 5 fuerzas de Porter (Rivalidad entre productores, Clientes, proveedores, sustitutos, Competencia potencial). Se contrastó 
el resultado de las evaluaciones agropecuarias departamentales (EVA) respecto a la información georeferenciada de aptitud de suelos disponible en el SIGAI-SENA, se realizó un inventario físico con GPS y analizó información secundaria proveniente de diferentes fuentes de información (SICMMA, ICA e IGAC).

El análisis permitió determinar el nivel de competitividad que presenta el municipio de El Carmen de Bolívar y su potencial agroindustrial desde el punto de vista de la producción agrícola y las capacidades productivas según clases agrológicas y aptitud de suelos.

\section{PRODUCCIÓN AGRICOLA DE EL CARMEN DE BOLÍVAR}

El Carmen de Bolívar es conocido comúnmente como la despensa agrícola y alimentaria del departamento de Bolívar. Este reconocimiento se debe a que gran parte de su producción agrícola se constituye en la dieta alimentaria de las poblaciones rurales y urbanas del departamento y la región Caribe Colombiana. Su producción agrícola se ha centrado, a lo largo del periodo 2000-2008, en trece (13) productos agrícolas, los cuales representaron casi el $50 \%$ de los cultivos producidos en la Zodes Montes de María. Durante este periodo, el promedio de áreas sembradas y cosechadas en el municipio fueron de 15.187 ha y $13.493 \mathrm{Ha}$, respectivamente. Cabe destacar que el 2008 fue un año de intensificación de cultivos, ya que las hectáreas sembradas y cosechadas se incrementaron, con respecto al 2007, en un 93,6 y $95,1 \%$, respectivamente.

Al analizar de manera detallada la estructura productiva agrícola, según los tipos de cultivos que tienen presencia en El Carmen de Bolívar, se observa que el $51,3 \%$ del área sembrada se concentra en cultivos anuales, dentro de los que se encuentra el ñame, el tabaco y la yuca. Los cultivos transitorios, por su parte, se convierten en la segunda clasificación productiva con mayor participación en el aparato productivo agrícola del municipio, con el 31,2\% del área total sembrada. Por último, los cultivos permanentes tales como el aguacate, el plátano y cacao, representan el 17,5\% del área agrícola sembrada del municipio (ver tabla 1). 
TABLA 1. ESTRUCTURA PRODUCTIVA DEL MUNICIPIO DEL CARMEN DE BOLÍVAR POR TIPO DE CULTIVO. AÑO 2008

\begin{tabular}{|c|c|c|c|c|c|c|}
\hline $\begin{array}{l}\text { Tipo De } \\
\text { Cultivos }\end{array}$ & $\begin{array}{c}\text { Área } \\
\text { Sembrada } \\
\text { (Has) }\end{array}$ & $\begin{array}{c}\text { Área } \\
\text { Cosechada } \\
\text { (Has) }\end{array}$ & $\begin{array}{l}\text { Producción } \\
\text { (Ton) }\end{array}$ & $\begin{array}{c}\text { \% Part. } \\
\text { Área } \\
\text { Sembrada } \\
\text { Total } \\
\text { Agrícola }\end{array}$ & $\begin{array}{c}\text { \% Part. } \\
\text { Área } \\
\text { Cosechada } \\
\text { Total } \\
\text { Agrícola }\end{array}$ & $\begin{array}{c}\text { \% Part. } \\
\text { Producción } \\
\text { Total } \\
\text { Agrícola }\end{array}$ \\
\hline $\begin{array}{l}\text { CULTIVOS } \\
\text { TRANSITO- } \\
\text { RIOS }\end{array}$ & 8.045 & 7.795 & 13.355 & $31,2 \%$ & $31,6 \%$ & $6,5 \%$ \\
\hline $\begin{array}{l}\text { CULTIVOS } \\
\text { PERMANEN- } \\
\text { TES }\end{array}$ & 4.500 & 3.700 & 44.400 & $17,5 \%$ & $15,0 \%$ & $21,5 \%$ \\
\hline $\begin{array}{l}\text { CULTIVOS } \\
\text { ANUALES }\end{array}$ & 13.200 & 13.200 & 148.500 & $51,3 \%$ & $53,5 \%$ & $72,0 \%$ \\
\hline TOTAL & 25.745 & 24.695 & 206.255 & $100,0 \%$ & $100,0 \%$ & $100,0 \%$ \\
\hline
\end{tabular}

Fuente: Elaboración de los autores con base a la Evaluación Agropecuaria del Departamento de Bolívar

En el año 2008, la producción agrícola de El Carmen de Bolívar estuvo compuesta por 11 productos de los que tradicionalmente se han cultivado en el territorio, en donde la yuca es el principal cultivo en la dinámica agrícola municipal, con un área sembrada total de 7.000 hectáreas, seguido por el Name con 6.000 hectáreas sembradas y el Maíz Blanco Tradicional con un total de 4.500 hectáreas sembradas (ver tabla 2).

TABLA 2. ESTRUCTURA PRODUCTIVA DEL MUNICIPIO DEL CARMEN DE BOLÍVAR POR CULTIVO. AÑO 2008

\begin{tabular}{|c|c|c|c|c|c|c|c|}
\hline Cultivo & $\begin{array}{c}\text { Área } \\
\text { Sembra- } \\
\text { da }\end{array}$ & $\begin{array}{l}\text { Área Co- } \\
\text { sechada }\end{array}$ & Producción & $\begin{array}{l}\text { Rendi- } \\
\text { miento }\end{array}$ & $\begin{array}{l}\mathbf{N}^{\circ} \text { pro- } \\
\text { ductores }\end{array}$ & $\begin{array}{l}\text { Participa- } \\
\text { ción }\end{array}$ & $\begin{array}{l}\text { Acumu- } \\
\text { lado }\end{array}$ \\
\hline YUCA & 7.000 & 7.000 & 76.000 & 10,0 & 9.000 & $27,2 \%$ & $27,2 \%$ \\
\hline NAME & 6.000 & 6.000 & 72.000 & 12,0 & 8.000 & $23,3 \%$ & $50,5 \%$ \\
\hline $\begin{array}{l}\text { MAIZ } \\
\text { BLANCO } \\
\text { TRADICIO- } \\
\text { NAL }\end{array}$ & 4.500 & 4.300 & 7.350 & 1,6 & 4.000 & $17,5 \%$ & $68,0 \%$ \\
\hline AGUACATE & 4.100 & 3.700 & 44.400 & 12,0 & 700 & $15,9 \%$ & $83,9 \%$ \\
\hline $\begin{array}{l}\text { MAIZ AMA- } \\
\text { RILLO TRA- } \\
\text { DICIONAL }\end{array}$ & 3.200 & 3.200 & 5.550 & 1,7 & 5.000 & $12,4 \%$ & $96,3 \%$ \\
\hline AJONJOLI & 300 & 250 & 200 & 0,8 & 450 & $1,2 \%$ & $97,5 \%$ \\
\hline TABACO & 200 & 200 & 500 & 2,5 & 400 & $0,8 \%$ & $98,3 \%$ \\
\hline CACAO & 200 & - & - & - & 90 & $0,8 \%$ & $99,0 \%$ \\
\hline PLATANO & 200 & - & - & - & 90 & $0,8 \%$ & $99,8 \%$ \\
\hline AJI DULCE & 30 & 30 & 165 & 5,5 & 50 & $0,1 \%$ & $99,9 \%$ \\
\hline $\begin{array}{l}\text { AJI TABAS- } \\
\text { CO }\end{array}$ & 15 & 15 & 90 & 6,0 & 20 & $0,1 \%$ & $100,0 \%$ \\
\hline TOTAL & 25.745 & & 206.255 & & 27.800 & $100 \%$ & \\
\hline
\end{tabular}

Fuente: Elaboración de los autores con base a la Evaluación Agropecuaria del Departamento de Bolívar 
Teniendo en cuenta las participaciones porcentuales que cada uno de los productos tiene en el área sembrada total del municipio para el año 2008, en la anterior tabla se observan los 5 productos agrícolas que concentran y absorben el 96,3\% del área sembrada. Estos productos totalizan 24.800 hectáreas agrícolas y se constituyen como los cultivos con mayor presencia y transcendencia en cuanto a extensión sembrada.

Los cultivos de Yuca, Name, Maíz Blanco Tradicional, Aguacate, y Maíz Amarillo Tradicional, conforman el grupo de productos, a los cuales, se le realizará el análisis de potencial agroindustrial para el municipio del Carmen de Bolívar, desde un enfoque de capacidades productivas.

\section{CAPACIDAD POTENCIAL DE USO DE SUELO PARA CULTIVOS AGRÍCOLAS DE EL CARMEN DE BOLÍVAR SEGÚN CLASES AGROLÓGICAS}

La clasificación de las tierras según su capacidad de uso es una metodología desarrollada por Klingebiel y Montgomery y estructurada por el Servicio de Conservación del Suelo de los Estados Unidos que ha sido ajustada por otros autores a través de los años, la cual interpreta a las características y propiedades intrínsecas del suelo, del medio físico y del nivel tecnológico de los agricultores, con el objeto de distinguir clases homogéneas de tierras basadas en el grado de limitación del uso, y definir su capacidad de uso potencial lo cual conduce a controlar la degradación de los suelos, especialmente la asociada a procesos de erosión acelerada (2).

Esta metodología se basa en un sistema que busca la producción máxima con base al aprovechamiento de las capacidades de los suelos con mínimas perdidas de su potencialidad. Para definir los tipos de uso de los suelos se definen cinco (5) sistemas de explotación agrícola: laboreo permanente, labores ocasionales, pastos, bosques y reservas naturales.

A su vez, el sistema define tres niveles de clasificación de los suelos, organizados en Clases, Subclases y Unidades. En este modelo se pueden identificar ocho (8) clases, en donde las limitaciones de uso van aumentando de manera creciente con cada una de las clases, obteniendo la clase I como la mejor hasta la clase VIII. Por otro lado, se pueden agrupar también las clases como arables (clases I a IV) y no arables (clase V a VIII). A continuación se explica cada una de las clases de suelos desarrolladas en este modelo (2). 
- Clase I: Esta clase presenta suelos ideales para el laboreo permanente o cualquier clase de explotación agrícola.

- Clase II: Suelos con buenas condiciones para laboreo permanente pero con algunas limitaciones.

- Clase III: Suelos con aceptables condiciones pero con severas limitaciones.

- Clase IV: suelos para laboreo ocasional o para pastos y bosques.

- Clase V, VI, VII: Suelos aptos solo para pastos o bosques (Reservas naturales), no es recomendable para usos agrícolas por presentar muy severas limitaciones y/o necesitar un alto y cuidadoso manejo.

- Clase VIII: Suelo apto solo para reservas naturales.

Basado en lo anterior, se puede identificar un primer grupo de tierras cultivables, aptas para la producción de cultivos anuales, permanentes, transitorios, pasturas y/o reforestación y/o vida silvestre; este grupo se compone por las clases I, II, III y IV. Un segundo grupo comprende tierras aptas para la producción de pasturas, reforestación y/o conservación de la vida silvestre, pero no son tierras aptas para realizar actividades que conduzcan a la realización de cultivos labrados comunes, este grupo comprende las clases V, VI y VII. Por último, encontramos tierras no aptas para cultivos, pero apropiadas para protección de la flora y de la fauna silvestre, recreación y almacenamiento de agua (clase VIII) (3).

Respecto a las limitaciones que se puede encontrar en las clases de suelos se establecen varias subclases de capacidad definidas por Klingebiel y Montgomery así:

- Subclase e, para riesgos de erosión.

- Subclase w, para problemas de hidromorfía.

- Subclase s, para limitaciones de suelos que afectan el desarrollo radicular.

- Subclase C, para limitaciones climáticas.

En el territorio de este municipio se pueden encontrar las clases agrológicas de tipo III, IV, VI y VII, con ciertas limitaciones como se pueden observar en la columna subclase en la tabla 4. La clase agrologica de mayor capacidad en cuanto a superficie disponible corresponde a la clase VI-es, con una superficie de $39.788,9$ hectáreas, equivalente al $44,2 \%$ del territorio municipal.

$\mathrm{Al}$ analizar las capacidades de cada una de las clases y contrastarlas con la realidad productiva del año 2008, se observa que la clase III-cs posee una capacidad de 10.781,54 hectáreas favorables para cultivos semestrales o 
transitorios como al maíz, al arroz, ají, el ajonjolí y para productos permanentes como el tabaco. A su vez, se observa que el área sembrada total para el año 2008 de los cultivos que tienen favorabilidad para la productividad en esta clase es de 8.245 hectáreas, determinándose así una utilización de esta clase agrologica del $76,5 \%$ de la capacidad total disponible (ver tabla 4).

\section{CAPACIDAD POTENCIAL DE USO DE SUELO PARA CULTIVOS AGRICOLAS DE EL CARMEN DE BOLIVAR SEGÚN APTITUD DE SUELO}

El modelo aplicado para el análisis de evaluación de tierras hace parte de las metodologías desarrolladas por la Organización de las Naciones Unidas para la Agricultura y la Alimentación - FAO (4), más específicamente, el método de máxima limitación. Este procedimiento define las cualidades del suelo y las clasifica en clases de aptitud, relacionándolas con la capacidad de generar rendimientos productivos y el uso de insumos o prácticas de manejo especificas para mejorar la tierra en función de lograr rendimientos por encima del óptimo deseado.

Para el análisis de capacidad basado en la aptitud de suelo disponible en la zona para el desarrollo de cultivos agrícolas, se diseñó una serie de mapas para los productos de mayor tradición y participación en la estructura productiva del municipio. De esta manera, se obtiene el área total disponible para cada cultivo según su aptitud y se compara con la estructura agrícola del municipio mediante un análisis aproximado de capacidades según su nivel de utilización, subutilización, o incluso, sobreutilización de los suelos disponibles.

A continuación se detalla la información de capacidades por cada uno de los cultivos que conforman la dinámica agrícola de El Carmen de Bolívar.

TABLA 3. APTITUD FÍSICA DE SUELOS PARA CULTIVOS EN EL CARMEN DE BOLÍVAR

\begin{tabular}{|c|c|c|c|c|c|c|c|}
\hline $\begin{array}{c}\text { Clase y } \\
\text { descripción }\end{array}$ & Yuca & Ñame & Maíz & Aguacate & Tabaco & Cacao & Plátano \\
\hline $\begin{array}{l}\text { Altamente Apto } \\
\text { Suelos óptimos de } \\
\text { poca inversión (A1) }\end{array}$ & $63.831,24$ & $56.440,89$ & $1.174,80$ & $6.105,21$ & $25.653,42$ & & $19.190,51$ \\
\hline $\begin{array}{l}\text { Medianamente Apto } \\
\text { Suelos viables con } \\
\text { prácticas de manejo } \\
\text { de mediana inversión } \\
\text { (A2) }\end{array}$ & & & $49.283,80$ & $24.761,65$ & & $29.547,13$ & $1.449,02$ \\
\hline $\begin{array}{l}\text { Marginalmente Apto } \\
\text { Suelos poco recomen- } \\
\text { dables por su alta } \\
\text { inversión y baja pro- } \\
\text { ductividad (A3) }\end{array}$ & $29.547,13$ & $36.937,48$ & $42.919,18$ & $62.511,52$ & $67.724,96$ & $63.831,24$ & $72.738,84$ \\
\hline
\end{tabular}

Fuente: Elaboración de los autores con base Centro de Información Geográfica Agropecuaria Integral-CIGAI 
TABLA 4. ANÁLISIS DE CAPACIDADES SEGÚN LAS CLASES AGROLÓGICAS DISPONIBLES EN EL MUNICIPIO DE EL CARMEN DE BOLÍVAR. 2008

\begin{tabular}{|c|c|c|c|c|c|c|c|}
\hline CLASE & $\begin{array}{l}\text { SUB- } \\
\text { CLASE }\end{array}$ & $\begin{array}{c}\text { USO Y PRACTICAS DE } \\
\text { MANEJO RECOMEN- } \\
\text { DADO }\end{array}$ & $\begin{array}{l}\text { Superfi- } \\
\text { cie Dis- } \\
\text { ponible }\end{array}$ & $\begin{array}{l}\text { Superfi- } \\
\text { cie utili- } \\
\text { zada }\end{array}$ & $\begin{array}{l}\text { Capaci- } \\
\text { dad } \\
\text { Utilizada }\end{array}$ & $\begin{array}{l}\text { Capacidad } \\
\text { Subutili- } \\
\text { zada o NO } \\
\text { utilizada }\end{array}$ & $\begin{array}{c}\text { Capaci- } \\
\text { dad } \\
\text { Sobre } \\
\text { explo- } \\
\text { tada }\end{array}$ \\
\hline III & CS & $\begin{array}{l}\text { Agricultura intensiva du- } \\
\text { rante un semestre (Cultivos } \\
\text { Transitorios): Maíz, Arroz Se- } \\
\text { cano, Ají, Ajonjolí, Tabaco y } \\
\text { posibilidad para adecuación } \\
\text { de Riego. Pastoreo controla- } \\
\text { do con rotación de potreros. }\end{array}$ & $10.781,54$ & 8.245 & $76,5 \%$ & $23,5 \%$ & - \\
\hline IV & $\mathrm{s}$ & $\begin{array}{l}\text { Áreas Aptas para cultivos } \\
\text { de Sorgo, Maíz, Algodón, } \\
\text { Ajonjolí, Tabaco, Cacao, Ag- } \\
\text { uacate, Ají y Yuca. Pastos in- } \\
\text { troducidos con buen manejo } \\
\text { agronómico para pastoreo } \\
\text { controlado. }\end{array}$ & $14.871,9$ & 11.300 & $76,0 \%$ & $24,0 \%$ & - \\
\hline IV & es & $\begin{array}{l}\text { Combinación de Cultivos } \\
\text { (Name, Yuca, Maíz, Plátano, } \\
\text { Frijol Caupí, Tabaco, Ajon- } \\
\text { jolí) con especies forestales. } \\
\text { Se debe favorecer la regen- } \\
\text { eración natural para evitar la } \\
\text { erosión. }\end{array}$ & $3.486,7$ & 6.200 & $177,8 \%$ & - & $77,8 \%$ \\
\hline VI & $\mathrm{s}$ & $\begin{array}{l}\text { Bosques productores/pro- } \\
\text { tectores, pastos adaptados a } \\
\text { suelos ácidos para pastoreo } \\
\text { controlado con prácticas de } \\
\text { conservación para evitar la } \\
\text { erosión. Frutales, Aguacate, } \\
\text { Plátano, Cacao con buen } \\
\text { manejo agronómico. }\end{array}$ & $5.350,8$ & - & $379,2 \%$ & - & $279,2 \%$ \\
\hline VI & es & $\begin{array}{l}\text { Frutales, Aguacate, Cacao, } \\
\text { Plátano, Caña Panelera, con } \\
\text { excelente manejo agronómi- } \\
\text { co para prevenir procesos } \\
\text { erosivos. Bosques produc- } \\
\text { tores / protectores, pastos. } \\
\text { En áreas de menor pendiente } \\
\text { bosque (Aguacate, Cacao, } \\
\text { Plátano) y pastos con prácti- } \\
\text { cas de conservación de Sue- } \\
\text { los para prevenir la erosión. }\end{array}$ & $39.788,9$ & 39.939 & $100,4 \%$ & $0,0 \%$ & - \\
\hline VII & es & $\begin{array}{l}\text { Unidades para bosque pro- } \\
\text { ductor o protector. En Áreas } \\
\text { de menor pendiente se pu- } \\
\text { eden cultivar frutales, Agua- } \\
\text { cate, Cacao, Plátano, Madera- } \\
\text { bles. No se recomienda la ac- } \\
\text { tividad Pecuaria bajo ningún } \\
\text { manejo. }\end{array}$ & $19.098,6$ & - & - & $100,0 \%$ & - \\
\hline
\end{tabular}

Fuente: Elaboración de los autores con base Centro de Información Geográfica Agropecuaria Integral-CIGAI 


\section{ANÁLISIS DE COMPETITIVIDAD DEL SECTOR AGRÍCOLA DE EL CARMEN DE BOLÍVAR}

Para construir el panorama de competitividad de la producción agrícola de El Carmen de Bolívar, se tomó como fuente de información las reuniones realizadas con los actores del municipio, como líderes de asociaciones productivas, empresarios de agronegocios y productores, como también los diagnósticos de competitividad realizados por la Comisión Regional de Competitividad de Cartagena y Bolívar y de los documentos resultado de las jornadas de trabajo del gobierno departamental y gobiernos locales para el diseño de las políticas y el Plan de Desarrollo de Bolívar 2008-2011 (5).

Dicho análisis se ha complementado con la información obtenida (e incluida en los capítulos anteriores) de la Secretaría de Educación de Bolívar, la Secretaría de Agricultura y Desarrollo rural de Bolívar, Universidad de Cartagena, SENA y demás documentos relacionados. Asimismo, se validó este diagnóstico mediante talleres de socialización y validación realizados en el municipio con la participación de productores agrícolas, dirigentes institucionales, propietarios de empresas agroindustriales, entre otros actores.

Los resultados arrojados son los que se presentan a continuación:

TABLA 5. VENTAJAS Y DESVENTAJAS DE LA PRODUCCIÓN AGRÍCOLA DE EL CARMEN DE BOLÍVAR DE ACUERDO A LOS COMPONENTES DEL DIAMANTE DE PORTER

\begin{tabular}{|c|c|}
\hline CONDICIONES DE FACTOR & $\begin{array}{c}\text { CONDICIONES DE LA } \\
\text { DEMANDA }\end{array}$ \\
\hline $\begin{array}{l}\text { Ventajas } \\
\text { - Conocimiento tácito en producción agrícola. } \\
\text { - Presencia de suelos con potencial de uso para la actividad agrícola. } \\
\text { - Localización geográfica estratégica por su cercanía con centros } \\
\text { urbanos de la región (Sincelejo, Montería, Barranquilla, Cartagena) } \\
\text { y del principal puerto del País. } \\
\text { - Distritos de riego con Potencial de uso. }\end{array}$ & $\begin{array}{l}\text { Ventajas } \\
\text {-Crisis alimentarias. } \\
\text {-Aumento en tendencias } \\
\text { internacionales en } \\
\text { alimentación orgánica y } \\
\text { productos naturales. } \\
\text {-Demanda local uniforme y } \\
\text { sin variaciones. }\end{array}$ \\
\hline $\begin{array}{l}\text { Desventajas } \\
\text {-Baja tecnificación e infraestructura productiva. } \\
\text {-Baja disponibilidad de recurso humano calificado especializado. } \\
\text { - Baja oferta, calidad y pertinencia educativa en temas relacionados } \\
\text { con las capacidades agrícolas de la región. } \\
\text { - Problema estructural y transversal de Agua en la región. } \\
\text {-Falta de proyección empresarial. } \\
\text { - Requisitos de Capital. } \\
\text { - Infraestructura Vial deficiente, especialmente, en la zona rural y } \\
\text { corregimientos productores. } \\
\text { - Resistencia a la aplicación de nuevas técnicas de producción y de } \\
\text { optimización de cultivos. } \\
\text { - Baja utilización de recursos logísticos disponibles en el municipio } \\
\text { - Subutilización de las capacidades de producción del municipio } \\
\text { (Suelos) }\end{array}$ & $\begin{array}{l}\text { Desventajas } \\
\text { - Poco conocimiento de } \\
\text { mercados a nacional e } \\
\text { internacional. } \\
\text { - Baja cultura a nivel local en } \\
\text { cuanto a productos agrícolas } \\
\text { con transformaciones } \\
\text { industriales. }\end{array}$ \\
\hline
\end{tabular}

Fuente: Elaboración de los autores 
TABLA 6. VENTAJAS Y DESVENTAJAS DE LA PRODUCCIÓN AGRÍCOLA DE EL CARMEN DE BOLÍVAR DE ACUERDO A LOS COMPONENTES DEL DIAMANTE DE PORTER

\begin{tabular}{|c|c|}
\hline ESTRATEGIA DE FIRMA Y RIVALIDAD & JADAS Y DE S \\
\hline $\begin{array}{l}\text { Ventajas } \\
\text {-Ventaja competitiva de la El Carmen de } \\
\text { Bolívar como principal productor agrícola } \\
\text { de Bolívar. } \\
\text {-Altos volúmenes de producción (Economía } \\
\text { de escala). }\end{array}$ & $\begin{array}{l}\text { Ventajas } \\
\text {-Alto volumen de producción de los proveedores } \\
\text { (productores) como sistema de producción. } \\
\text {-Disponibilidad de materia prima como insumo del } \\
\text { modelo de producción agroindustrial. }\end{array}$ \\
\hline $\begin{array}{l}\text { Desventajas } \\
\text {-Baja integración y sinergia entre los actores } \\
\text { del sistema productivo. } \\
\text { - Baja diferenciación en los productos. } \\
\text { - Baja rivalidad en la dinámica interna del } \\
\text { sector. } \\
\text { - Escasas empresas de tipo agroindustrial. } \\
\text { - Ausencia de productos con alta capacidad } \\
\text { productiva de la El Carmen de Bolívar en las } \\
\text { agendas de competitividad departamental. } \\
\text {-Alta Exigencia en cuanto a normas y } \\
\text { requisitos para exportación de productos } \\
\text { agrícolas. } \\
\text {-Baja rentabilidad en el sector. } \\
\text {-Alto nivel de informalidad en la actividad } \\
\text { agrícola en la Zona. } \\
\text {-Baja productividad. } \\
\text {-Pequeñas extensiones de las unidades } \\
\text { agrícolas. }\end{array}$ & $\begin{array}{l}\text { Desventajas } \\
\text { Falta de consolidación de las cadenas productivas } \\
\text { Bajo poder de negociación de los productores. } \\
\text { Falta de apoyo de la institucionalidad en el sector en } \\
\text { materia de apoyo, investigación y desarrollo. } \\
\text { Falta de articulación entre la política pública, } \\
\text { gobernabilidad y las capacidades productivas de la } \\
\text { región. } \\
\text { Inefectividad en el manejo de los recursos públicos } \\
\text { municipales. } \\
\text { Baja inversión en educación para fortalecer las } \\
\text { capacidades humanas del municipio. }\end{array}$ \\
\hline
\end{tabular}

Fuente: Elaboración de los autores

\section{CONCLUSIONES}

Más allá de cualquier factor condicionante de la competitividad, el conocimiento adquirido e implícito en cuanto a las prácticas de producción agrícola por parte de los agricultores, se convierte en un factor importante a la hora de determinar la capacidad del municipio. Este know-how se basa en todos los conocimientos y practicas adquiridas por los agricultores a través de la práctica agrícola inherente en la tradición general de la población de El Carmen de Bolívar.

Mediante este estudio se identifica el potencial de la producción agrícola en cuanto a la disponibilidad de los suelos en el municipio El Carmen de Bolívar según sus capacidades de uso y aptitud de suelos, encontrando que a nivel municipal, se encuentran clases agrologicas de tipo III y IV recomendadas para la actividad agrícola siempre que se les aplique una rotación de cultivos adecuada o un tratamiento pertinente y otras clases que, aunque con menor capacidad de uso, también presentan condiciones para la producción de productos agrícolas como frutales, aguacate, entre otros. 
En la dinámica de los sectores agrícolas a nivel de países en desarrollo, la agricultura concentra las poblaciones más pobres de los países, y en parte es debido, a la baja productividad que se refleja en bajos ingresos para sus productores. Esta baja productividad se debe principalmente a dos factores: Las pequeñas extensiones destinadas a la producción por cada unidad productiva y la poca o ninguna utilización de tecnología moderna, lo cual incide, en una desventaja a la hora de competir con países con sectores agrícolas más desarrollados.

En este estudio se evidenció la poca o nula presencia de programas académicos de las instituciones educativas presente en la región, encontrando una baja pertinencia de las instituciones educativas, principalmente a nivel de educación universitaria. Por otro lado, es común encontrar que, por la falta de oportunidades de desarrollo para los jóvenes de las regiones rurales, se observa una migración hacia las ciudades, perdiéndose así, potencialidades de capital humano para el futuro.

Uno de los principales problemas que aquejan los productores en El Carmen de Bolívar es la baja integración entre los diferentes actores del sistema, ya que, a pesar de los esfuerzos de instituciones como la fundación Montes de María (6) y de el III Laboratorio de Paz, aún se evidencian los escasos flujos de información y la baja integración de las unidades productivas (Visión individual y no de Clústeres).

Elmodelo de competitividad plantea que en todo mercadoesindispensable un nivel de rivalidad en su dinámica interna que permita que haya un mejoramiento de las capacidades de los productores basado en la innovación de sus procesos. En la dinámica productiva de El Carmen de Bolívar no se evidencia un nivel de rivalidad y una cultura empresarial que exija un mejoramiento de los procesos de producción existente.

No se evidencian cadenas productivas consolidadas e integradas en El Carmen de Bolívar para los principales productos del municipio.

Existe una ausencia de institucionalidad en su función de apoyar en el desarrollo de la región, especialmente de las instituciones educativas, de investigación y de desarrollo. 


\section{REFERENCIAS BIBLIOGRÁFICAS}

1. Girán, Rainer, Vargas, Humberto y Osta, Karelys. Propuesta metodológica para el análisis de competitividad empresarial en la pequeña y mediana agroindustria alimentaria. 1, Carabobo Valencia (Venezuela) : Escuela de Ingeniería - Facultad de Ingeniería Universidad de Carabobo, 2008, Revista Ingeniería Industrial, Vol. 7.

2. Dorronsoro, Carlos. Sistemas de evaluación de capacidades de uso categóricos. Granada - España : Universidad de Granada Departamento de Edafología y Química Agrícola, 2008. Tercer curso de ciencias ambientales, gestión y conservación de Aguas.

3. Zelay, K. y Cabria, F. Descripción de la Aptitud de las Tierras en el Área de Influencia del Centro Regional Buenos Aires Sur. 12, Buenos Aires : Instituto Nacional de Tecnología Agropecuaria, 2007, Vol. II.

4. Organización de las Naciones Unidas para la Alimentación y la Agricultura - FAO. Evaluación de tierras con metodología de FAO Proyecto regional Ordenamiento Territorial Rural Sostenible. Santiago de Chile : Naciones Unidas, 2003.

5. ComisiónRegionalCompetitividad deCartagenay Bolívar. Diagnóstico y diseño de estrategias para el incremento de la competitividad del departamento de Bolívar. Cartagena de Indias : s.n., 2007.

6. Fundación Red Desarrollo y Paz de los Montes de María. Socialización Proyecto de Fortalecimiento de Cadenas Productivas por parte de la Coporación PBA. San Juan Nepomuceno - Bolívar : s.n., 2010.

\section{BIBLIOGRAFÍA RECOMENDADA}

BENZAQUEN, J., del CARPIO, L. A., ZEGARRA, L. A. y VALDIVIA, C. A., (2010), Un Índice Regional de Competitividad para un país., Revista CEPAL 102. p69-86.

PÉREZ-CASTAÑO B. J., (2007), Competitividad, desarrollo e ingeniería: algunas definiciones y reflexiones, Revista Ingeniería y Competitividad, Volumen 9, No. 1, p. 57 - 75.

ROMERO, S., (1999), Territorio, agricultura y competitividad., IICA, San José, Costa Rica. 
